\title{
Ácido Hipocloroso: una Nueva Alternativa como Agente Antimicrobiano y para la Proliferación Celular para Uso en Odontología
}

\author{
Hypochlorous Acid: A New Alternative as Antimicrobial Agent \\ and For Cell Proliferation for Use in Dentistry
}

Gloria Inés Lafaurie*; Justo Leonardo Calderón”; Carlos Zaror ${ }^{* * *}$; Lina Viviana Millán ${ }^{* * *}$ \& Diana Marcela Castillo ${ }^{* * * *}$

LAFAURIE, G. I.; CALDERÓN, J. L.; ZAROR, C.; MILLÁN, L. V. \& CASTILLO, D. M. Ácido hipocloroso: una nueva alternativa como agente antimicrobiano y para la proliferación celular para uso en odontología. Int. J. Odontostomat., 9(3):475-481, 2015.

RESUMEN: El ácido hipocloroso $(\mathrm{HOCl})$ es un potente antimicrobiano no antibiótico utilizado en medicina clínica para el control de infecciones y reparación de heridas. In vivo el $\mathrm{HOCl}$ es sintetizado por células del sistema inmune para el control del agente patógeno durante la fagocitosis y ha sido sintetizado y estabilizado en el laboratorio con potenciales aplicaciones profilácticas y terapéuticas en medicina humana. El efecto antimicrobiano, antinflamatorio y en la proliferación celular lo hacen una sustancia que debe ser más evaluada para uso clínico en otras áreas de salud. Existe un interés en el desarrollo de nuevas sustancias antimicrobianas de uso tópico en odontología para el control del biofilm dental, la inflamación gingival y para la cicatrización de heridas de la mucosa oral. Se presenta una revisión de la literatura de los principales efectos del $\mathrm{HOCl}$ que sustentan su investigación y uso en odontología.

PALABRAS CLAVE: acido hipocloroso, $\mathrm{HOCl}$, biofilm dental, cicatrización de heridas.

\section{INTRODUCCIÓN}

La cavidad oral provee un microambiente ideal para el crecimiento de múltiples especies bacterianas y dentro de la flora oral se pueden encontrar especies residentes que componen la flora normal, así como bacterias transeúntes y otras asociadas a enfermedad. La placa dental es uno de los tipos de biofilm más estudiados y es la forma de crecimiento más frecuente de las bacterias de la cavidad oral (Palmer, 2009). Múltiples estudios han comprobado que la eliminación mecánica del biofilm dental de manera controlada, reduce significativamente la cantidad de la misma y procesos de gingivitis (van der Weijden \& Hioe, 2005). Sin embargo, para mejorar su reducción y de la inflamación gingival se han desarrollado múltiples sustancias antiplaca. Teniendo en cuenta que la flora normal mantiene en equilibrio el ecosistema oral y reduce la posibilidad de infecciones por patógenos exógenos, se puede decir que estos tratamientos deben apuntar a controlar más que a erradicar la placa dental (Slots \& Jorgensen, 2002; Palmer). La clorhexidina ha sido la sustancia más estudiada en la inhibición del biofilm dental y es altamente utilizada para protocolos de desinfección completa de la boca (Eberhard et al., 2008) y su efecto sobre la reducción de gingivitis ha sido bien documentado (Van Strydonck et al., 2012). Aunque la clorhexidina no parece ser más

\footnotetext{
Odontóloga, Especialista en Periodoncia, Profesor titular, Directora Unidad de Investigación Básica Oral-UIBO, Facultad de Odontología, Universidad El Bosque, Bogotá, Colombia.

* Bacteriólogo, Director científico Aquilabs SA, Bogotá, Colombia.

*** Departamento de Odontopediatría y Ortodoncia, CIEESPO, Facultad de Odontología, Universidad de la Frontera, Temuco, Chile.

**** Bacterióloga, Magister en Microbiología, Profesor Asistente, Investigador Unidad de Investigación Básica Oral-UIBO, Facultad de Odontología, Universidad El Bosque, Bogotá, Colombia.

Bartorterióloga, Magister en Microbiología, Profesor Asociado, Investigador Unidad de Investigación Básica Oral-UIBO, Facultad de Odontología, Universidad El Bosque, Bogotá, Colombia.

Fuente de Financiamiento: Esta investigación fue financiada por el Instituto Colombiano de Ciencia y Tecnología "Francisco José de Caldas" COLCIENCIAS (código 130850227678) Contrato 774-2011.
} 
efectivo como agente anti gingivitis que otros enjuagues como los aceites esenciales cuando se compara su uso a largo plazo, sigue siendo el agente más efectivo cuando el objetivo de la terapia es la reducción del biofilm dental (Van Leeuwen et al., 2011; Neely, 2012). A pesar de su amplia utilización, algunos efectos colaterales han desestimulado el uso clínico de la clorhexidina y estos incluyen: pigmentación dental, ya que se mezcla fácilmente con los cromógenos de la dieta; escasa actividad microbicida a bajas concentraciones y en altas concentraciones puede producir dermatitis; lesión de mucosas y resequedad de los tejidos, situación que altera y retrasa el proceso de cicatrización (Jones, 1997; Silvestri \& McEneryStonelake, 2013; Graziani et al., 2015).

En los últimos años se ha aumentado el interés por nuevas moléculas de alta potencia con efecto antiplaca con bioequivalencia con la clorhexidina pero con menos efectos adversos. El ácido hipocloroso es propuesto como agente antiplaca y para la cicatrización de heridas de la mucosa oral por su baja toxicidad, su comprobada efectividad antimicrobiana, su capacidad antinflamatoria e inductora de la proliferación celular y por sus antecedentes como sustancia de uso tópico en desinfección de heridas en medicina (Selkon et al., 2006; Sam \& Lu, 2009).

El propósito de esta revisión es presentar los principales mecanismos de acción del $\mathrm{HOCl}$ que sustentan su investigación para uso clínico en odontología.

Ácido hipocloroso (HOCl). Los usos terapéuticos del $\mathrm{HOCl}$ inician en la Primera Guerra Mundial con los estudios de Alexis Carrel y Henry Dakin quienes obtuvieron una solución de hipoclorito de sodio acidificado y tamponado (solución de Dakin), el cual generaba concentraciones ideales de $\mathrm{HOCl}$, la cual fue utilizada exitosamente para desinfección de heridas (Levine, 1991). La solución de Dakin modificada a una concentración del 0,025\% mostró ser terapéuticamente efectiva como apósito en el manejo de heridas ya que preserva las propiedades terapéuticas con la eliminación del efecto tóxico potencial en la cicatrización de heridas (Heggers et al., 1991). Este procedimiento fue conocido como la técnica de Carrel-Dakin y se convirtió en el método para tratar las heridas infectadas durante la guerra (Cusimano et al., 1984)

El HOCl biológicamente, se clasifica dentro de un grupo de pequeñas moléculas conocidas como especies reactivas del oxígeno (ROS) sintetizadas por células del sistema inmune (neutrófilos y macrófagos) en un proceso inmunológico conocido como "estallido respiratorio", durante la fagocitosis de antígenos (Weiss, 1989). Cuando un patógeno invade los tejidos, los polimorfonucleares neutrofilos y macrófagos son activados y la fagocitosis de los antígenos es el resultante del ensamblaje e inicio del estallido respiratorio, mediado por la NADPH oxidasa en la membrana del fagolisosoma. A su vez grandes cantidades de oxígeno molecular son utilizadas para producir radicales libres. $\mathrm{NADPH}$ oxidasa, induce una reducción de oxígeno molecular $\left(\mathrm{O}_{2}\right)$ catalizado en la membrana del fagosoma produciendo el anión superóxido $\left(\cdot \mathrm{O}_{2}^{-}\right)$. $\mathrm{El} \mathrm{O}_{2}{ }^{-}$es una molécula muy inestable que se dismuta espontáneamente o por acción de la enzima superóxido dismutasa, para producir peróxido de hidrógeno $\left(\mathrm{H}_{2} \mathrm{O}_{2}\right)$. Este complejo enzimático vincula compuestos halogenados plasmáticos como es el caso del cloro $(\mathrm{Cl}-)$. Debido a que la concentración de cloro en el plasma es mil veces mayor que la de otros compuestos halogenados, el sistema $\mathrm{H}_{2} \mathrm{O}_{2}-$ mieloperoxidasa utiliza el cloro formando $\mathrm{HOCl}$ quien es altamente reactivo en contra de componentes esenciales de la célula microbiana (LekstromHimes \& Gallin, 2000; Mainnemare et al., 2004) (Fig.1). $\mathrm{EI} \mathrm{HOCl}$, resultante del estallido respiratorio, se libera entonces a nivel extracelular reaccionando con la taurina formando cloramina de taurina un oxidante de larga duración que también tiene propiedades antibacteriana y antinflamatoria (Sam \& Lu).

Obtención. El HOCl es un ión no disociado del cloro dependiente del oxígeno, altamente inestable y altamente reactivo. Por ser uno de los ácidos hipo halogenados más fuertes, es también uno de los más poderosos oxidantes entre los oxácidos clorados y es el responsable directo de la acción bactericida de los compuestos derivados del cloro (Weiss). Químicamente el $\mathrm{HOCl}$ puede ser obtenido por diferentes métodos. Infortunadamente, la mayoría de los procedimientos obtienen soluciones de baja estabilidad, moderada actividad microbicida, con bajo porcentaje de ácido hipocloroso libre.

In vitro el $\mathrm{HOCl}$ se puede obtener a través de tres diferentes métodos:

1. Hidrólisis de gas de cloro $\left(\mathrm{Cl}_{2}+\mathrm{H}_{2} \mathrm{O}^{-}>\mathrm{HOCl}+\mathrm{H}^{+}\right.$ $\left.+\mathrm{Cl}^{-}\right)$

2. Electrolisis de solución de sal $\left(2 \mathrm{Cl}^{-}+2 \mathrm{e}^{-}>\mathrm{Cl}_{2}\right)$ y $\left(\mathrm{Cl}_{2}+\mathrm{H}_{2} \mathrm{O}>\mathrm{HOCl}+\mathrm{H}^{+}+\mathrm{Cl}^{-}\right)$

3. Acidificación de hipoclorito $\left(\mathrm{OCl}^{-}+\mathrm{H}^{+}, \mathrm{HOCl}\right)$ : Debido a que el hipoclorito está disponible comercial- 


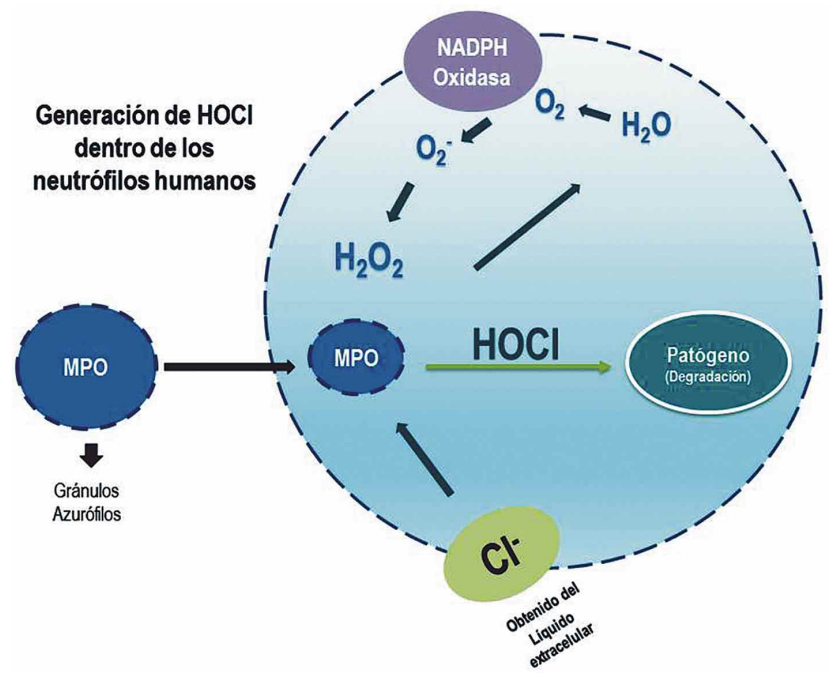

Fig. 1. Representación esquemática de la producción de $\mathrm{HOCl}$ a nivel intracelular durante el estallido respiratorio. La célula utiliza el oxígeno molecular $\left(\mathrm{O}_{2}\right)$ para producir peróxido de hidrógeno $\left(\mathrm{H}_{2} \mathrm{O}_{2}\right)$ utilizando la enzima NADPH oxidasa. La enzima mieloperoxidasa (MPO) depositada en los gránulos azurófilos cataliza la reacción $\mathrm{H}_{2} \mathrm{O}_{2}$ y cloro $(\mathrm{Cl})$ para formar ácido hipocloroso $(\mathrm{HOCl})$. La reacción toma lugar en el lumen del fagolisosoma el cuál se va acidificando a medida que el proceso avanza, estabilizando y optimizando la actividad antimicrobiana molécula de $\mathrm{HOCl}$.

mente, este método es el más utilizado, conveniente y controlable.

1. Hidrólisis de gas de cloro: consiste en la aplicación de gas cloro directamente en el agua. Este método es muy utilizado en procesos de desinfección de aguas para piscinas, acueductos e industria. Sin embargo, los usos clínicos son limitados, tanto por las altas concentraciones de especies cloradas en solución, como también por la inestabilidad del producto final (Monarca et al., 2004; Lowe et al., 2013)

2. Electrolisis de sales: comercialmente el método de electrólisis de sales y agua es muy utilizado. Permite la formación de especies cloradas y $\mathrm{HOCl}$ in situ para su uso en un tiempo no mayor a 6 horas, generando formulaciones con $\mathrm{HOCl}$ ideales para procesos de desinfección de superficies, dispositivos médicos tales como endoscopios, equipos quirúrgicos, entre otros. Por otro lado, múltiples compañías han patentado diferentes métodos de electrólisis para la generación de "agua súper-oxidada" con fines médicos. Este método consiste en la utilización de una celda electroquímica, compuesta de cátodos y ánodos que transmiten un pulso eléctrico a una mezcla homogénea de agua y buffers salinos. La carga eléctrica permite el incremento del potencial oxidativo del agua (ORP >1000 mV). El fenómeno eléctrico también permite la dismutación de las sales y la posterior liberación de especies cloradas en solución, entre las que se encuentran: $\mathrm{NaOCl}, \mathrm{NaCl}$ en su mayoría y $\mathrm{HOCl}$ en un menor porcentaje (concentraciones variables de $\mathrm{HOCl}$ que van desde $2 \mathrm{ppm}$ hasta $100 \mathrm{ppm}$ ) pero considerándose el ingrediente activo de las formulaciones obtenidas a través de este sistema (Innoue et al., 1997; Landa-Solis et al., 2005)

3. Acidificación de hipoclorito: debido a que el hipoclorito está disponible comercialmente, este método es el más utilizado, conveniente y controlable. Permite la mayor generación de $\mathrm{HOCl}$ en solución, con un potencial redox entre $1000 \mathrm{mV}-1200 \mathrm{mV}$. Infortunadamente en múltiples casos las soluciones obtenidas carecen de la estabilidad necesaria para su uso prolongado (Wang et al., 2007).

En Colombia en 1993, a través de la implementación de un protocolo modificado de acidificación más procesos secundarios de súper oxidación del agua, se desarrolla una técnica para la estabilización de $\mathrm{HOCl}$ para su uso en medicina $(\mathrm{HOCl}$ en buffer salino, con un ORP de $1200 \mathrm{mV}-1320 \mathrm{mV}$, y a un $\mathrm{pH}$ ajustado de 5,2, sin sólidos en solución y con una densidad de $1,0( \pm 0,1) \mathrm{g} / \mathrm{m}$ a concentraciones que van desde 0,1 ppm hasta $7000 \mathrm{ppm}$ ). Este desarrollo dio inicio a procesos investigativos dirigidos a la optimización y refinamiento de los métodos de obtención de la molécula hasta desarrollar una solución antimicrobiana a base de $\mathrm{HOCl}$ con potenciales aplicaciones profilácticas y terapéuticas en medicina humana, aprobada por el instituto de vigilancia en alimentos y medicamentos de Colombia (INVIMA) (Riveros et al., 2003; Naranjo et al., 2006). En 2006, la FDA (Food and Drug Administration) en los EE.UU aprobó una solución cuyo componente activo es el ácido hipocloroso; el Aquatine (Sterilox Dental's Aquatine Endodontic Cleanser, Aquatine EC, Sterilox, Puricore, Malvern, PA, USA) fue aprobado para ser usado como irrigante en endodoncia siendo otro producto patentado para uso en odontología (García et al., 2010).

Efecto Antimicrobiano del $\mathrm{HOCl}$. El interés por el $\mathrm{HOCl}$ hizo que se desarrollaran técnicas in vitro para generar $\mathrm{HOCl}$. Análisis cuantitativos demostraron que al activar 1X106 neutrófilos se producen aproximadamente 2X10-7 mol de $\mathrm{HOCl}$ durante una incubación de dos horas, esta cantidad es capaz de destruir 150 millones de Escherichia coli (Weiss). 
El ácido hipocloroso es el componente activo del hipoclorito de sodio sin sus efectos adversos, de esta manera, se podría considerar como un potente antiplaca para uso en cavidad oral ya que se ha demostrado un alto efecto antimicrobiano (Gray et al., 2013). El HOCl ha demostrado tener un efecto antimicrobiano de amplio espectro en concentraciones que van desde 0,1 a $2,8 \mathrm{mg} / \mathrm{ml}$ en un periodo de exposición de $2 \mathrm{~min}$. Esta actividad microbicida, a pesar de ser más efectiva para formas bacterianas que para esporas y hongos, y abarca microorganismos clínicamente relevantes como lo son bacterias Gram negativas, Gram positivas, parásitos y hongos (Wang et al.).

Las bacterias Gram negativas contienen grupos sulfuro y hemo (ricos en hierro) en su membrana externa que son esenciales para llevar a cabo normalmente el transporte de electrones. Una reacción enzimática irreversible de $\mathrm{HOCl}$ con proteínas de membrana, produce daños estructurales que alteran la permeabilidad celular y afecta la viabilidad bacteriana (Rosen \& Klebanoff, 1982; Mckenna \& Davies, 1988). En bacterias Gram positivas, el HOCl actúa sobre los grupos aminos de la glicina presente en el peptidoglucano, por lo que la cloración en este grupo de microorganismos difiere en cuanto al blanco de acción. Sin embargo, la acción antimicrobiana del HOCl es mayor para microorganismos Gram negativos que para la flora Gram positiva (Sam \& Lu). El HOCl oxida y/o clorina endotoxinas y exotoxinas, neutralizando su acción y también oxida residuos de cisteína en gingipaínas como Rgp y Kgp. Además, $\mathrm{HOCl}$ interfiere en el componente C5 de la cascada del complemento que al activarse produce dos fracciones, entre ellas la C5b con actividad lítica sobre la membrana celular bacteriana. También promueve una respuesta inmune innata sobre bacterias Gram negativas vía cloración de antígenos, sin embargo, la cloración de bacterias Gram positivas y los antígenos liberados de estas, afectan significativamente la actividad secretora de citocinas producidas por macrófagos tales como el óxido nítrico y TNF alfa (Marcinkiewicz et al., 1994). Lo anterior indica que el $\mathrm{HOCl}$ estaría más indicado para el control de placa en pacientes con altos niveles de bacterias anaerobias y debería ser más dirigido a pacientes con enfermedad periodontal.

\section{Efecto anti-inflamatorio y sobre la proliferación} tisular del $\mathrm{HOCl}$. El $\mathrm{HOCl}$ tiene propiedades proinflamatorias y antinflamatorias que son dosis dependiente. A bajas dosis el $\mathrm{HOCl}$ puede activar proformas de metaloproteinasas (MMPs), colagenasas y gelatinasas. En altas concentraciones el $\mathrm{HOCl}$ inhibe la MMP-7, la actividad de la colagenasa y de la gelatinasa (Fu et al., 2003). Sin embargo, la acción antinflamatoria parece ser predominante aunque estas acciones deben ser evaluadas in vivo (Sam \& Lu). El $\mathrm{HOCl}$ tiene la capacidad de oxidar el aminoácido taurina e inducir la formación de cloro-taurina la cual posee actividad antimicrobiana de amplio espectro y larga duración y es la principal responsable del efecto antimicrobiano del $\mathrm{HOCl}$. La cloro-taurina tiene un importante efecto protector sobre los tejidos, ya que puede inhibir la producción de mediadores inflamatorios tales como anión superóxido, óxido nítrico, interleucinas y prostaglandinas en las células inflamatorias del tejido afectado contribuyendo de esta forma con los procesos de protección tisular (Kim \& Cha, 2014.) El HOCl también puede inhibir las vías de señalización vinculadas con la expresión y traslocación de factores de transcripción por oxidación como el factor nuclear kb. El factor nuclear kb es un importante transductor de señal responsable de la trascripción genética de muchos mediadores de la inflamación como IL-1a, IL-1b, IL-2, IL-6, TNF- $\partial$, NO, prostaglandina E2, TGF-ß y moléculas de adhesión, así como inhibidores de apoptosis. Todos los mediadores de inflamación mencionados con anterioridad están relacionados con la periodontitis, y su inhibición fisiológica parece ser fundamental en el proceso de recuperación del tejido periodontal (Sam \& Lu).

El $\mathrm{HOCl}$ produce un efecto estimulante en la proliferación celular, durante traumatismos o daño a tejidos, mediado por el estímulo en la producción de factores de crecimiento como el factor de crecimiento epidermal (EGF), factor de crecimiento de fibroblastos (FGF-1, FGF-2), factor de crecimiento del endotelio vascular y factor de crecimiento de tejido conectivo (Grzesik \& Narayanan, 2002). Igualmente, el HOCl presenta una importante actividad oxidativa sobre el péptido del extremo N-terminal del TGF-B lo que facilita el acceso del $\mathrm{HOCl}$ al sitio activo de la molécula, resultando en la activación del factor, promoviendo proliferación y fibrosis (Schrementi et al., 2008; Sam \& Lu).

Estudios en medicina clínica del $\mathrm{HOCl}$. El HOCl ha sido evaluado como antiséptico en desinfección de heridas de piel. El $\mathrm{HOCl}$ obtenido por electrólisis de sales ha mostrado ser efectivo en eliminación de esporas persistentes de Bacillus anthracis y Clostridium difficile en manos y piel (Nerandzic et al., 2013). La utilidad del ácido hipocloroso también ha sido evaluada en el tratamiento de ulceras y lesiones de piel que tienden a hacer a una lenta y deficiente cicatrización 


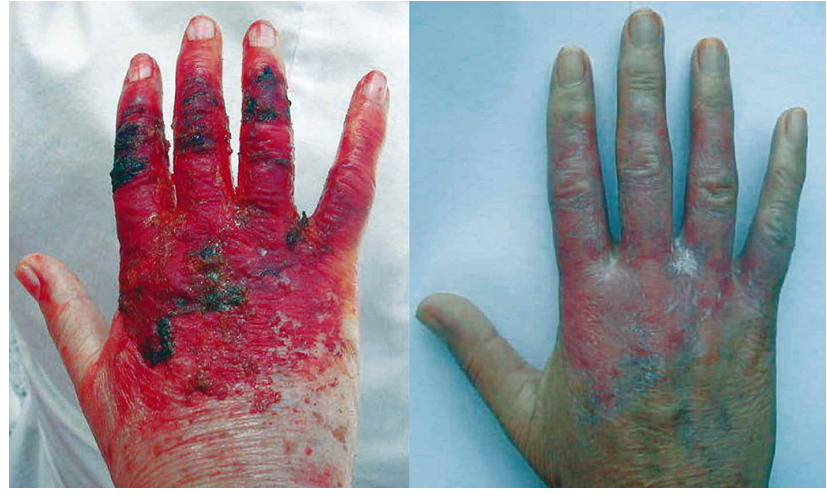

Fig. 2. Paciente con quemadura tratado con $\mathrm{HOCl}$ (Neutroderm ${ }^{\circledR}$ ) antes y después de 10 días de tratamiento mediante vendaje oclusivo con la solución.

(Fig. 2). Selkon et al., evaluaron clínicamente una versión de $\mathrm{HOCl}$ en el tratamiento de úlceras crónicas de miembros inferiores. El estudio reveló que los lavados del área afectada con $\mathrm{HOCl}$ permiten un apreciable incremento en el tejido de granulación y disminución del dolor. Naranjo et al., tratando la misma patología, pudieron concluir que la aplicación de $\mathrm{HOCl}$ tres veces al día sobre el área afectada, acompañada de vendaje oclusivo, facilita y acelera el proceso de regeneración de tejidos asociado al control microbiano local, al generar un microambiente húmedo ideal para el tejido de granulación.

Usos en odontología. Soluciones de $\mathrm{HOCl}$ han sido desarrolladas para uso odontológico para irrigación en endodoncia. El Aquatine (irrigante a base de $\mathrm{HOCl}$ ) ha sido comparado con el hipoclorito de sodio y la efectividad antimicrobiana y de remoción de barrillo dentinario del Aquatine mostró un comportamiento similar al del hipoclorito de sodio al $6 \%$ cuando fueron acompañados con una irrigación con EDTA. Sin embargo, el Aquatine fue superior al hipoclorito de sodio en términos de biocompatibilidad, convirtiéndose en una alternativa segura para la irrigación y desinfección de los conductos radiculares (García et al.). Ring et al. (2008) realizaron un estudio in vitro para determinar el efecto del irrigante de uso endodóntico en la adherencia celular a las paredes dentinales del conducto radicular en endodoncia regenerativa. Después de evaluar 10 soluciones para irrigación de conductos radiculares encontraron que la cantidad de células stem de pulpa dental (DPSCs) variaba de acuerdo a la solución irrigante y quelante utilizada, observando que en promedio, las cifras más altas de DPSCs unidas al conducto radicular eran identificadas cuando se utilizaba la solución irrigante Aquatine y EDTA, y el promedio más bajo fue cuando se usó $\mathrm{NaOCl}$ y MTA.
Uno de los intereses del estudio del $\mathrm{HOCl}$ radica en que este es la parte activa de hipoclorito de sodio con menos efectos adversos. El hipoclorito de sodio ha sido utilizado en algunos estudios de inhibición de microorganismos subgingivales en bolsas periodontales (Galván et al., 2013; Gonzalez et al., 2015). Concentraciones de hipoclorito de sodio al $0.05 \%$ también ha sido utilizado en enjuague bucal para la reducción de la gingivitis en un período de 21 días. Sin embargo, el hipoclorito de sodio presenta algunos efectos colaterales que incluyen irritación de las membranas mucosas cuando se usa a altas concentraciones, efectos decolorantes y efectos corrosivos de algunos metales (De Nardo et al., 2012). Dado que el $\mathrm{HOCl}$ es la parte activa y no tóxica de hipoclorito de sodio, su efecto sobre los principales microorganismos del biofilm dental han sido evaluados por nuestro grupo. El HOCl logró inhibición bacteriana para bacterias del biofilm dental a una concentración de $0,05 \%$ a 1 min para Streptococcus sanguis, Streptococcus mutans, Porphyromonas gingivalis, Eikenella corrodens, Campylobacter rectus, Fusobacterium nucleatum, y para microorganismos sobre infectantes como Enterococcus faecalis, Enterobacter cloacae, Klebsiella oxytoca y Klebsiella pneumoniae. Los resultados de nuestros estudios indican que el ácido hipocloroso es una alternativa antimicrobiana para bacterias con capacidad patogénica de cavidad oral. Candida albicans mostró inhibición a una concentración de $0,05 \%$ a 10 minutos lo que indica más bajo poder anti fúngico. A una concentración del $0,025 \%$ el $\mathrm{HOCl}$ mostró una inhibición parcial de las bacterias Gram positivas y bacterias entéricas evaluadas (Lafaurie et al., 2009).

Recientemente, se desarrollaron soluciones de $\mathrm{HOCl}$ a 250 y 500 ppm a un $\mathrm{pH}$ de 5,8 para ser utilizado en investigación para uso odontológico. Actualmente, se están realizando pruebas microbiológicas, de citotoxicidad sobre células de la mucosa oral, efectos del $\mathrm{pH}$ sobre la capacidad buffer de la saliva, efecto erosivo sobre el esmalte dental, su efecto sobre la oxidación de proteínas y estudios de sustantividad y efecto antiplaca que sustenten su uso en cavidad oral como agente antimicrobiano y en cicatrización de heridas (Proyecto COLCIENCIAS, 2011).

\section{CONCLUSIONES}

Dada la obtención y estabilización de soluciones a base de $\mathrm{HOCl}$ para uso clínico y por su biocompatibilidad y los estudios preliminares de su 
acción antimicrobiana sobre los microorganismos de la biofilm dental, los mecanismos anti-inflamatorios y sus efectos sobre la proliferación tisular, abren nuevos campos de estudio del $\mathrm{HOCl}$ como agente antiplaca y en la reducción de la inflamación gingival especialmente en pacientes con periodontitis, en endodoncia regenerativa y en la reparación de heridas de uso en odontología.

LAFAURIE, G. I.; CALDERÓN, J. L.; ZAROR, C.; MILLÁN, L. V. \& CASTILLO, D. M. Hypochlorous acid: A new alternative as antimicrobial agent and for cell proliferation for use in dentistry. Int. J. Odontostomat., 9(3):475-481, 2015.

ABSTRACT: Hypochlorous acid $(\mathrm{HOCl})$ is a powerful non antibiotic antimicrobial solution used in clinical medicine for infection control and wound healing. In vivo $\mathrm{HOCl}$ is produced by cells of the immune system to control the pathogen during phagocytosis and has been synthesized and stabilized in the laboratory with potential prophylactic and therapeutic applications in human medicine. The antimicrobial, anti-inflammatory and cell proliferation effect make it a substance to be evaluated in other health areas. There is interest to development of new antimicrobial substances for use in oral health for the control of dental biofilm, gingival inflammation and wound healing of the oral mucosa. A review of the literature of the main effects of $\mathrm{HOCl}$ that support its research and use in dentistry is presented.

KEY WORDS: hypochlorous acid, $\mathrm{HOCl}$, dental biofilm, wound healing.

\section{REFERENCIAS BIBLIOGRAFÍCAS}

Cusimano, R.; Cusimano, M. \& Cusimano, S. The genius of Alexis Carrel. Can. Med. Assoc. J., 131(9):1142-50, 1984.

De Nardo, R.; Chiappe, V.; Gómez, M.; Romanelli, H. \& Slots, J. Effects of $0.05 \%$ sodium hypochlorite oral rinse on supragingival biofilm and gingival inflammation. Int. Dent. J., 62(4):208-12, 2012.

Eberhard, J.; Jepsen, S.; Jervøe-Storm, P. M.; Needleman, I. \& Worthington, H. V. Full-mouth disinfection for the treatment of adult chronic periodontitis. Cochrane Database Syst. Rev., (1):CD004622, 2008.

Fu, X.; Kassim, S. Y.; Parks, W. C. \& Heinecke, J. W. Hypochlorous acid generated by myeloperoxidase modifies adjacent tryptophan and glycine residues in the catalytic domain of matrix metalloproteinase-7 (matrilysin): an oxidative mechanism for restraining proteolytic activity during inflammation. J. Biol. Chem., 278(31):28403-9, 2003.

Garcia, F.; Murray, P. E.; Garcia-Godoy, F. \& Namerow, K. N. Effect of Aquatine Endodontic Cleanser on smear layer removal in the root canals of ex vivo human teeth. J. Appl. Oral Sci., 18(4):403-8, 2010.

Galván, M.; Gonzalez, S.; Cohen, C. L.; Alonaizan, F. A.; Chen, C. T.; Rich, S. K. \& Slots, J. Periodontal effects of $0.25 \%$ sodium hypochlorite twice-weekly oral rinse. A pilot study. J. Periodontal Res., 49(6):696-702, 2013.

Gonzalez, S.; Cohen, C. L.; Galván, M.; Alonaizan, F. A.; Rich, S. K. \& Slots, J. Gingival bleeding on probing: relationship to change in periodontal pocket depth and effect of sodium hypochlorite oral rinse. J. Periodontal Res., 50(3):397-402, 2015.

Gray, M. J.; Wholey, W. Y. \& Jakob, U. Bacterial responses to reactive chlorine species. Annu. Rev. Microbiol., 67:141-60, 2013.
Graziani, F.; Gabriele, M.; D'Aiuto, F.; Suvan, J.; Tonellie, M. \& Cei, S. Dental plaque, gingival inflammation and tooth discolouration with different commercial -formulations of $0.2 \%$ chlorhexidine rinse: a double-blind randomised controlled clinical trial. Oral Health. Prev. Dent., 13(2):10111,2015

Grzesik, W. J. \& Narayanan, A. S. Cementum and periodontal wound healing and regeneration. Crit. Rev. Oral Biol. Med., 3(6):474-84, 2002.

Heggers, J. P.; Sazy, J. A.; Stenberg, B. D.; Strock, L. L.; McCauley, R. L.; Herndon, D. N. \& Robson, M. C. Bactericidal and wound-healing properties of sodium hypochlorite solutions: the 1991 Lindberg Award. J. Burn Care Rehabil., 12(5):420-4, 1991.

Inoue, Y.; Endo, S.; Kondo, K.; Ito, H.; Omori, H. \& Saito, K. Trial of electrolyzed strong acid aqueous solution lavage in the treatment of peritonitis and intraperitoneal abscess. Artif. Organs, 21(1):28-31, 1997.

Jones, C. G. Chlorhexidine: is it still the gold standard? Periodontol. 2000, 15:55-62, 1997.

Kim, C. \& Cha, Y. N. Taurine chloramine produced from taurine under inflammation provides anti-inflammatory and cytoprotective effects. Amino Acids, 46(1):89-100, 2014.

Landa-Solis, C.; González-Espinosa, D.; Guzmán-Soriano, B.; Snyder, M.; Reyes-Terán, G.; Torres, K. \& Gutierrez, A. A. Microcyn: a novel super-oxidized water with neutral $\mathrm{pH}$ and disinfectant activity. J. Hosp. Infect., 61(4):291-9, 2005.

Lafaurie, G. I.; Aya, M. R.; Arboleda, S.; Escalante, A.; Castillo, D. M.; Millán, L. V.; Calderón, J. \& Ruiz, B. N. Eficacia desinfectante del ácido hipocloroso sobre cepas con poder patogénico de cavidad oral. Rev. Colomb. Investig. Odontol., 1(1):3-11, 2009. 
Lekstrom-Himes, J. A. \& Gallin, J. I. Immunodeficiency diseases caused by defects in phagocytes. N. Engl. J. Med., 343(23):1703-14, 2000.

Levine, J. M. Dakin's solution: past, present, and future. Adv. Skin Wound Care, 26(9):410-4, 2013.

Lowe, J. J.; Gibbs, S. G.; Iwen, P. C.; Smith, P. W. \& Hewlett, A. $\mathrm{L}$. Impact of chlorine dioxide gas sterilization on nosocomial organism viability in a hospital room. Int. J. Environ. Res. Public Health, 10(6):2596-605, 2013.

Mainnemare, A.; Mégarbane, B.; Soueidan, A.; Daniel, A. \& Chapple, I. L. Hypochlorous acid and taurine-Nmonochloramine in periodontal diseases. J. Dent. Res., 83(11):823-31, 2004.

Mckenna, S. M. \& Davies, K. J. The inhibition of bacterial growth by hypochlorous acid. Possible role in the bactericidal activity of phagocytes. Biochem. J., 254(3):685-92, 1988.

Monarca, S.; Zani, C.; Richardson, S. D.; Thruston, A. D. Jr.; Moretti, M.; Feretti, D. \& Villarini, M. A. A new approach to evaluating the toxicity and genotoxicity of disinfected drinking water. Water Res., 38(17):3809-19, 2004.

Marcinkiewicz, J.; Czajkowska, B.; Grabowska, A.; Kasprowicz, A. \& Kociszewska, B. Differential effects of chlorination of bacteria on their capacity to generate NO, TNF-alpha and IL-6 in macrophages. Immunology, 83(4):611-6, 1994.

Naranjo, J.; Acevedo, C. \& Calderón, J. L. Uso del ácido hipocloroso en ulceras de miembros inferiores. Inf. Med., 94:8-11, 2006.

Neely, A. L. Essential oil mouthwash (EOMW) may be equivalent to chlorhexidine $(\mathrm{CHX})$ for long-term control of gingival inflammation but $\mathrm{CHX}$ appears to perform better than EOMW in plaque control. J. Evid. Based Dent. Pract., 12(3 Suppl.):69-72, 2012.

Nerandzic, M. M.; Rackaityte, E.; Jury, L. A.; Eckart, K. \& Donskey, C. J. Novel strategies for enhanced removal of persistent Bacillus anthracis surrogates and Clostridium difficile spores from skin. PloS One, 8(7):e68706, 2013.

Palmer, R. J. Oral bacterial biofilms--history in progress. Microbiology, 155(Pt. 7):2113-4, 2009.

Proyecto COLCIENCIAS. Código 130850227678. Contrato 7742011. Evaluación del ácido hipocloroso para uso en cavidad oral. Bogotá, Instituto Colombiano de Ciencia y Tecnología "Francisco José de Caldas" COLCIENCIAS, 2011.

Ring, K. C.; Murray, P. E.; Namerow, K. N.; Kuttler, S. \& GarciaGodoy, F. The comparison of the effect of endodontic irrigation on cell adherence to root canal dentin. J. Endod., 34(12):1474-9, 2008.

Riveros, S. C. H.; Parada, C. R. S.; Alvarez, J. A. G. Actividad bactericida del ácido hipocloroso. Rev. Fac. Med. Univ. Nac. Colomb., (51)3:136-42, 2003.
Rosen, H. \& Klebanoff, S. J. Oxidation of Escherichia coli iron centers by the myeloperoxidase-mediated microbicidal system. J. Biol. Chem., 257(22):13731-5, 1982.

Sam, C. H. \& Lu, H. K. The role of hypochlorous acid as one of the reactive oxygen species in periodontal disease. J. Dent. Sci., 4(2):45-54, 2009.

Schrementi, M. E.; Ferreira, A. M.; Zender, C. \& DiPietro, L. A Site-specific production of TGF-beta in oral mucosal and cutaneous wounds. Wound Repair Regen., 16(1):80-6, 2008.

Selkon, J. B.; Cherry, G. W.; Wilson, J. M. \& Hughes, M. A. Evaluation of hypochlorous acid washes in the treatment of chronic venous leg ulcers. J. Wound Care, 15(1):33-7, 2006.

Silvestri, D. L. \& McEnery-Stonelake, M. Chlorhexidine: uses and adverse reactions. Dermatitis, 24(3):112-8, 2013.

Slots, J. \& Jorgensen, M. G. Effective, safe, practical and affordable periodontal antimicrobial therapy: where are we going, and are we there yet? Periodontol. 2000, 28:298-12, 2002.

van der Weijden, G. A. \& Hioe, K. P. A systematic review of the effectiveness of self-performed mechanical plaque removal in adults with gingivitis using a manual toothbrush. J. Clin. Periodontol., 32 (Suppl. 6):214-28, 2005.

Van Leeuwen, M. P.; Slot, D. E. \& Van der Weijden, G. A. Essential oils compared to chlorhexidine with respect to plaque and parameters of gingival inflammation: a systematic review. J. Periodontol., 82(2):174-94, 2011.

Van Strydonck, D. A.; Slot, D. E.; Van der Velden, U. \& Van der Weijden, F. Effect of a chlorhexidine mouthrinse on plaque, gingival inflammation and staining in gingivitis patients: a systematic review. J. Clin. Periodontol., 39(11):1042-55, 2012.

Wang, L.; Bassiri, M.; Najafi, R.; Najafi, K.; Yang, J.; Khosrovi, B.; Hwong, W.; Barati, E.; Belisle, B.; Celeri, C. \& Robson, M. C. Hypochlorous acid as a potential wound care agent: part I. Stabilized hypochlorous acid: a component of the inorganic armamentarium of innate immunity. J. Burns Wounds, 6:e5, 2007.

Weiss, S. J. Tissue destruction by neutrophils. N. Engl. J. Med., 320(6):365-76, 1989.

\section{Dirección para Correspondencia:}

Gloria Lafaurie

Profesor titular

Coordinadora Unidad de Investigaciones

Facultad de Odontología

Universidad El Bosque

Recibido : 21-11-2014

Aceptado: 01-10-2015

Email: institutouibo@gmail.com 